\title{
Three-Dimensional Spoiled Gradient MRI
}

National Cancer Institute

\section{Source}

National Cancer Institute. Three-Dimensional Spoiled Gradient MRI. NCI Thesaurus. Code C118463.

A magnetic resonance imaging modality that uses acquisition in the steady state with semi-random changes in the phase of the radio frequency pulses to produce a spatially independent phase shift. This optimizes the signal-to-noise ratio to provide high resolution and a decrease in the partial volume phenomenon. 